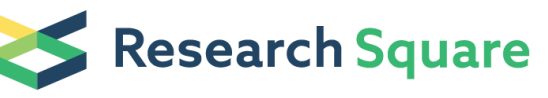 \\ Preprints are preliminary reports that have not undergone peer review. \\ They should not be considered conclusive, used to inform clinical practice, or referenced by the media as validated information.
}

\section{LncRNA CYTOR Regulates Chondrocyte Apoptosis in Osteoarthritis by Acting as a CeRNA for miR-10a- $5 p$}

Jiayi Luo

The Affiliated Hospital of Guizhou Medical University

Cheng Wu ( $\sim$ ChengWuHospital@163.com )

The Affiliated Hospital of Guizhou Medical University

Yuanxin Liu

The Affiliated Hospital of Guizhou University

Jiannan Fan

affiliated hospital of Guizhou University

\section{Xianwen Shang}

The Affiliated Hospital of Guizhou Medical University

\section{Riguang Liu}

Affiliated Hospital of Guiyang Medical University: The Affiliated Hospital of Guizhou Medical University

\section{Chuan Ye}

The Affiliated Hospital of Guizhou Medical University

Jihong Yang

The Affiliated Hospital of Guizhou Medical University

Hong Cao

The Affiliated Hospital of Guizhou Medical University

\section{Research}

Keywords: osteoarthritis, CYTOR, miR-10a-5p, ceRNA

Posted Date: October 23rd, 2020

DOl: https://doi.org/10.21203/rs.3.rs-95416/v1

License: (c) (i) This work is licensed under a Creative Commons Attribution 4.0 International License.

Read Full License 


\section{Abstract}

Background: It has been reported that IncRNA CYTOR suppresses LPS-induced inflammation, which plays crucial roles in osteoarthritis (OA). This study was therefore performed to analyze the role of CYTOR in OA.

Methods: Expression of CYTOR and miR-10a-5p in OA patients and chondrocytes as well as healthy patients and chondrocytes was determined by RT-qPCR. The interaction between CYTOR and miR-10a-5p was predicted by IntaRNA online program and confirmed by dual luciferase activity assay. The role of CYTOR and miR-10a-5p in regulating the apoptosis of chondrocytes were analyzed by cell apoptosis assay.

Results: CYTOR was under-expressed in OA patients and chondrocytes, and miR-10a-5p was overexpressed in OA patients and chondrocytes. After treatment, CYTOR was upregulated and miR-10a$5 p$ was downregulated. CYTOR was predicted to interact with miR-10a-5p, and the interaction between them was confirmed by dual luciferase reporter assay. Overexpression of CYTOR and miR-10a-5p failed to affect the expression of each other. Cell apoptosis analysis showed that CYTOR overexpression reduced the enhancing effects of miR-10a-5p overexpression on the apoptosis of OA chondrocytes.

Conclusion: CYTOR may suppress the development of OA by serving as a ceRNA for miR-10a-5p.

\section{Background}

Osteoarthritis (OA), also known as degenerative joint disease, is the most common form of arthritis that mainly affects the protective cartilage around joints [1]. The highest prevalence of OA is observed in the age group $>60$ years, it is estimated that more than $13 \%$ of females and $10 \%$ of males in this age group are suffering for $\mathrm{OA}[2,3]$. At present, there is no cure for $\mathrm{OA}$ [4]. Current therapeutic approaches main focus on the relief of symptoms, such as the use of Acetaminophen to reduce mild to moderate pain [5]. However, medications may not be helpful for severe cases. In addition, long-term use of Acetaminophen may cause liver damage [6]. Therefore, novel therapies are needed.

$\mathrm{OA}$ is closely correlated with aging [7]. Besides that, the development and progression of OA also require the involvement of molecular pathways, such as Notch and NF-KB signaling [8]. With the increased elucidation of the molecular mechanism of $O A$, novel therapies, such as targeted therapy to treat $O A$ by regulating gene expression, have been developed $[9,10]$. However, safe and effective treatments for $O A$ remain lacking. LncRNAs are not directly responsible for protein-coding but they regulate gene expression network to participate in human diseases including $O A$, suggesting that they are potential targets for $O A$ treatment [11]. However, the function of most IncRNAs in OA remains to be elucidated. In a recent study, IncRNA CYTOR was reported to play critical roles in LPS-induced cell injury [12], which can promote OA development [13]. We performed RNA-RNA interaction and found that CYTOR could interact with miR-10a$5 p$, which may promote the apoptosis of miR-10a-5p to participate in OA [14]. We then explore the interactions between CYTOR and miR-10a-5p in OA. 


\section{Results}

\section{Expression of CYTOR and miR-10a-5p was altered in both OA patients and chondrocytes.}

Expression of CYTOR and miR-10a-5p in OA patients (synovial fluid) and chondrocytes as well as healthy patients (synovial fluid) and chondrocytes was determined by RT-qPCR. Compared to controls, CYTOR was significantly under-expressed in OA patients and chondrocytes (Fig. 1A, p < 0.01). In contrast, miR$10 a-5 p$ was significantly overexpressed in OA patients and chondrocytes compared to corresponding controls (Fig. 1B, $p$ < 0.01). Therefore, altered expression of CYTOR and miR-10a-5p may participate in OA.

\section{Treatment increased the expression of CYTOR and decreased the expression of miR-10a-5p in OA patients}

Expression of CYTOR and miR-10a-5p in synovial fluid collected from OA patients before and 1 and 2 months after the initiation of treatment. Heatmaps were plotted using Heml 1.0 software to present the changes in the expression levels of CYTOR and miR-10a-5p during treatment. Compared to pre-treatment levels, CYTOR was upregulated and miR-10a-5p was downregulated during treatment.

\section{CYTOR and miR-10a-5p directly interacted with each other in two types of chondrocytes}

The potential interaction between CYTOR and miR-10a-5p was predicted by IntaRNA2.0. It was observed that CYTOR and miR-10a-5p may form basepairing with each other (Fig. 3A). Dual luciferase reporter assay was performed to confirm the interaction between CYTOR and miR-10a-5p. Compared to the CYTOR luciferase vector + NC miRNA group (NC group), luciferase activity was significantly lower in CYTOR luciferase vector + miR-10a-5p mimic group (miR-10a-5p group), suggesting the direct interaction between them (Fig. 3B, $p<0.05$ ).

\section{Overexpression of CYTOR and miR-10a-5p failed to alter the expression of each other}

To further explore the interaction between CYTOR and miR-10a-5p, OA and control chondrocytes were transfected with either CYTOR expression vector or miR-10a-5p mimic, and the expression of them was detected by RT-qPCR every $24 \mathrm{~h}$ until $96 \mathrm{~h}$. It was observed that CYTOR and miR-10a-5p were significantly overexpressed between $48 \mathrm{~h}$ and $96 \mathrm{~h}$ (Fig. 4A, p < 0.05). However, at different time points, overexpression of CYTOR failed to significantly affect the expression of miR-10a-5p (Fig. 4B, p<0.05), and overexpression of miR-10a-5p also showed no significant effect on the expression of CYTOR (Fig. 4C). Therefore, CYTOR is unlikely a target of miR-10a-5p.

\section{CYTOR overexpression suppressed the apoptosis of OA chondrocytes through miR-10a-5p}

The role of CYTOR and miR-10a-5p in regulating the apoptosis of OA and control chondrocytes was analyzed by cell apoptosis assay. CYTOR overexpression decreased the apoptosis of OA chondrocytes, and miR-10a-5p overexpression increased OA chondrocytes. Moreover, CYTOR overexpression reduced 
the enhancing effects of miR-10a-5p overexpression on the apoptosis of OA chondrocytes (Fig. 5A, $p<$ 0.05). In contrast, overexpression of CYTOR and miR-10a-5p failed to significantly affect the expression of control chondrocytes (Fig. 5B). Therefore, CYTOR may suppress the apoptosis of OA chondrocytes by serving as a ceRNA of miR-10a-5p.

\section{Discussion}

The involvement of CYTOR in OA and its interaction with miR-10a-5p were analyzed in this study. We found that CYTOR was downregulated in OA and overexpression of CYTOR may suppress the apoptosis of OA chondrocytes by serving as a ceRNA of miR-10a-5p.

CYTOR has been characterized as an oncogenic IncRNA in many types of cancers $[15,16]$. In cancers, CYTOR is overexpressed to promote cancer development by increasing cell proliferation and suppressing cell apoptosis $[15,16]$. In this study we found that CYTOR was downregulated in both OA patients and OA chondrocytes. Moreover, overexpression of CYTOR decreased the apoptosis of OA chondrocytes.

Therefore, CYTOR may play protective roles in OA, and overexpression of CYTOR may serve as a target for the treatment of OA. However, in vivo animal model experiments and clinical trials are needed to test our hypothesis. It is also worth noting that CYTOR suppress cell apoptosis in both cancers $[15,16]$ and OA. Therefore, CYTOR may play similar roles in cell apoptosis in different human diseases.

It has been reported that miR-10a-5p is overexpressed in OA and targets HOXA1 to promote the apoptosis of chondrocytes [14]. Consistently, we also observed the overexpression of miR-10a-5p in OA and its enhancing effects on the apoptosis of OA chondrocyte. However, the upstream regulator or miR-10a-5p in OA is unclear. Our study showed that PTV1 and miR-10a-5p could interact with each other, while they failed to affect the expression of each other. Moreover, CYTOR overexpression suppressed the role of miR-10a-5p in enhancing OA chondrocyte apoptosis. Therefore, CYTOR may suppress the apoptosis of OA chondrocytes by serving as a ceRNA of miR-10a-5p.

Interestingly, CYTOR and miR-10a-5p could directly interact with each other in both OA and control chondrocytes, while they only regulate the apoptosis of OA chondrocytes, but not control chondrocytes. Therefore, other pathological factors may interact with CYTOR/miR-10a-5p to participate in the apoptosis of chondrocytes in $\mathrm{OA}$.

\section{Conclusion}

In conclusion, CYTOR is under-expressed in OA and may promote the apoptosis of OA chondrocytes by serving as the ceRNA of miR-10a-5p.

\section{Materials And Methods}

OA patients and healthy controls 
This study enrolled 52 OA patients (20 males and 32 females, severe stages) and 52 healthy controls (20 males and 32 females) at Affiliated Hospital of Guizhou Medical University between March 2019 and June 2020. This study was approved by Ethics Committee of this hospital. Age ranges of patients and control were both 52 to 66 years, with a median of 59 years. All OA patients were diagnosed for the first time and no severe clinical disorders were diagnosed. Initiated therapy was excluded from the patients. All healthy controls showed normal physiological functions during systemic physiological health test. All patients signed informed consent. According to affected sites, the 52 patients included 20 cases of knee, 14 cases of hip, and 18 cases of feet.

\section{Treatment and synovial fluid preparation}

Synovial fluid (about $1.0 \mathrm{ml}$ ) was extracted from the affected sites of patients and the corresponding sites of controls prior to therapy. All patents were treated with acetaminophen and physical therapy. Synovial fluid (about $1.0 \mathrm{ml}$ ) was also extracted from the affected sites of patients at 1 and 2 months after the initiation of therapy. All synovial fluid samples were kept in liquid nitrogen storage prior to subsequent assays.

\section{Two types of chondrocytes}

Chondrocytes derived from an adult OA patient (4020A-05A) and a healthy control (402-05A) were both from Sigma-Aldrich. Chondrocyte growth medium (PromoCell) was used to cultivate cells at $37^{\circ} \mathrm{C}, 5 \% \mathrm{CO}_{2}$ and $95 \%$ humidity.

\section{Transfections and dual luciferase reporter assay}

CYTOR expression vector was constructed using pcDNA3.1 vector (Invitrogen) as backbone. Mimic of miR-10a-5p and miRNA negative control (NC) were the products from Sigma-Aldrich. Two types of chondrocytes were transfected with either $1 \mu \mathrm{g}$ CYTOR expression vector or $50 \mathrm{nM}$ miRNA using lipofectamine 2000 (Invitrogen). NC cells (empty vector- or NC miRNA- transfected cells) and Control (C, untransfected cells) cells were included.

CYTOR luciferase vector was established with pGL3 luciferase vector (Promega) as backbone. Lipofectamine 2000 (Invitrogen) was used to co-transfect CYTOR luciferase vector+NC miRNA (NC group) or CYTOR luciferase vector+miR-10a-5p mimic (miR-10a-5p group), and luciferase activity was measured at $48 \mathrm{~h}$ later.

\section{RNA isolation and process}

Trizol reagent (Invitrogen) was used to isolate total RNA from both synovial fluid samples and chondrocytes, followed by genomic DNA removal performed by incubating with DNase I (Invitrogen) for 2h. Electrophoresis performed using 5\% urea-PAGE gels was used to check RNA integrity. OD 260/280 ratios were determined to reflect RNA purity and a ratio close to 2.0 indicates pure RNA. 


\section{RT-qPCRs}

PrimeScript RT Reagent Kit (Takara Bio) was used for the preparation of cDNA samples with total RNA samples as template. QuantiTect SYBR Green PCR Kit (QIAGEN) was used to perform all qPCRs with 18S rRNA as an internal control to analyze the expression of CYTOR. Expression of miR-10a-5p was analyzed by The All-in-One ${ }^{\mathrm{TM}}$ miRNA qRT-PCR Detection Kit (Genecopoeia) with U6 as an internal control. Each PCR was performed in three technical replicates and $2^{-\Delta \Delta C t}$ method was used to normalize $C t$ values of target genes to corresponding internal controls.

\section{Cell apoptosis assay}

Two types of chondrocytes were subjected to cell apoptosis assay at 48h post-transfection. In a 6-well cell culture plate, chondrocytes $(20,000$ cells in $2 \mathrm{ml}$ medium per well) were cultivated for further $48 \mathrm{~h}$. After that, cells were washed with ice-cold PBS and resuspended in binding buffer. Following Annexin-V FITC and propidium iodide $(\mathrm{PI})$ staining, FACSCalibur instrument was used to analyze cell apoptosis.

\section{Statistical analysis}

Unpaired t test was used to compare the expression levels of CYTOR and miR-10a-5p between patients and controls. Heatmaps were plotted using Heml 1.0 software to present the changes in the expression levels of CYTOR and miR-10a-5p during treatment. Data of cell transfection experiments was expressed as mean $\pm S D$ values of three biological replicates and ANOVA Tukey's test was used for data comparison. $P<0.05$ was statistically significant.

\section{Declarations}

\section{Ethics approval and consent to participate}

This study was approved by Ethics Committee of Affiliated Hospital of Guizhou Medical University.

\section{Consent for publication}

Not applicable

\section{Availability of data and materials}

The datasets used analyzed during the current study are available from the corresponding author on reasonable request.

\section{Competing interests}

The authors declare that they have no competing interests.

\section{Funding}


This work was supported by Guiyang Science and Technology Bureau Numbering $\mathbb{\mathbb { 2 }}[2018] 1-79$

\section{Authors' contributions}

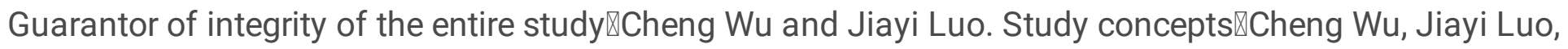
and Chuan Ye. Study design囚Jiayi Luo and Jiannan Fan. Definition of intellectual content $\bigotimes$ Xianwen Shang, Riguang Liu, and ChuanYe. Literature research囚Jihong Yang and Xianwen Shang. Clinical studies $\bigotimes$ Jiayi Luo, Cheng Wu, and Yuanxin Liu. Experimental studies $\mathbb{C}$ Cheng Wu, Jiayi Luo, and Yuanxin Liu. Data

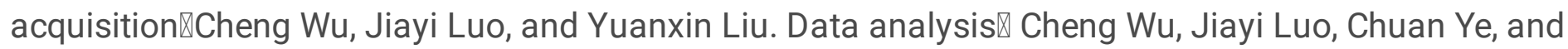
Jiannan Fan. Statistical analysis $₫$ Chuan Ye and Cheng Wu. Manuscript preparation囚Jiayi Luo, Cheng Wu, and Jihong Yang. Manuscript editing $₫$ Jiayi Luo, Cheng Wu, Chuan Ye, Rigaung Liu, Xianwen Shang, and

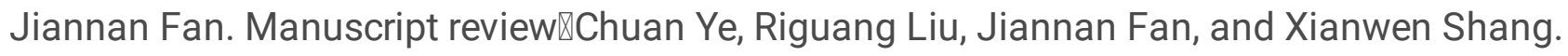

\section{Acknowledgements}

The authors would like to express our gratitude for those who have critically reviewed this manuscript and those who give us help during this experiment.

\section{References}

1. Glyn-Jones S, Palmer AJR, Agricola R, Price AJ, Vincent TL, Weinans $\mathrm{H}$, et al. Osteoarthritis. Lancet. 2015; 386(9991): 376-387.

2. Hügle T. Update Osteoarthritis. Rev Med Suisse. 2020; 16(685): 500-502.

3. Mandl LA. Osteoarthritis year in review 2018: clinical. Osteoarthr Cartilage. 2019; 27(3): 359-364.

4. Sinusas K. Osteoarthritis: diagnosis and treatment. Am Fam Physician. 2012; 85(1): 49-56.

5. Siebert DM, Meyer MC, Jensen E. Acetaminophen for Pain Relief in Osteoarthritis. Am Fam Physician. 2020; 102(2): 113-114.

6. Rahmati M, Nalesso G, Mobasheri A, Mozafari M. Aging and osteoarthritis: Central role of the extracellular matrix. Ageing Res Rev. 2017; 40: 20-30.

7. Chen D, Shen J, Zhao W, Wang T, Han L, Hamilton JL et al. Osteoarthritis: toward a comprehensive understanding of pathological mechanism. Bone Res. 2017; 5:16044.

8. Saito T, Tanaka S. Molecular mechanisms underlying osteoarthritis development: Notch and NF-kB. Arthritis Res Ther. 2017; 19(1):94.

9. de Campos GC, Tieppo AM, de Almeida Jr CS, Hamdan PC, Alves WM, de Rezende MU. Target-based approach for osteoarthritis treatment. World J Orthop. 2020; 11(6): 278-284.

10. Conaghan PG, Cook AD, Hamilton JA, Tak PP. Therapeutic options for targeting inflammatory osteoarthritis pain. Nat Rev Rheumatol. 2019; 15(6): 355-363.

11. Wu Y, Shen B, Zeng Y. The therapeutic potential and role of miRNA, IncRNA, and circRNA in osteoarthritis. Curr Gene Ther. 2019; 19(4): 255-263. 
12. Chen T, Zhu C, Ye C. LncRNA CYTOR attenuates sepsis-induced myocardial injury via regulating miR24/XIAP. Cell Biochem Funct. 2020; doi: 10.1002/cbf.3524.

13. Zhao LR, Xing RL, Wang PM, Zhang NS, Yin SJ, Li XC, et al. NLRP1 and NLRP3 inflammasomes mediate LPS/ATP-induced pyroptosis in knee osteoarthritis. Mol Med Rep. 2018; 17(4): 5463-5469.

14. Ma Y, Wu Y, Chen J, Huang K, Ji B, Chen Z, et al. miR-10a-5p promotes chondrocyte apoptosis in osteoarthritis by targeting HOXA1. Mol Ther Nucleic Acids. 2019; 14: 398-409.

15. Liu Y, Li M, Yu H, Piao H. IncRNA CYTOR promotes tamoxifen resistance in breast cancer cells via sponging miR-125a-5p. Int J Mol Med. 2020; 45(2): 497-509.

16. Zhu H, Shan Y, Ge K, Lu J, Jia C. LncRNA CYTOR promotes pancreatic cancer cell proliferation and migration by sponging miR-205-5p. Pancreatology. 2020; S1424-3903(20)30155-1.

\section{Figures}


Patients

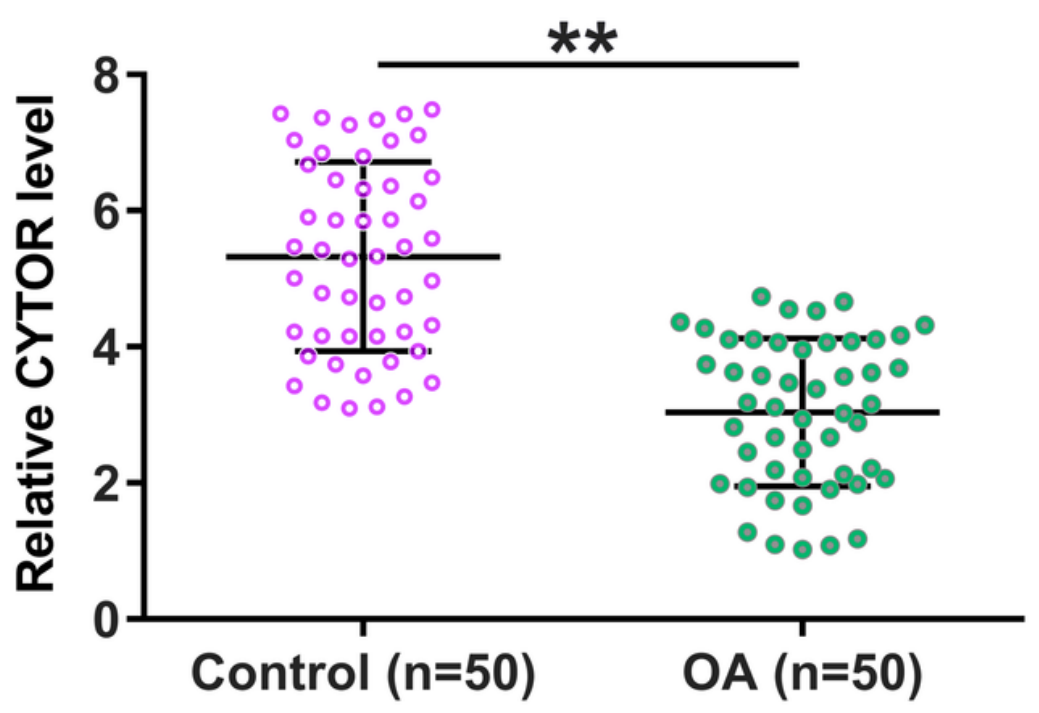

Patients

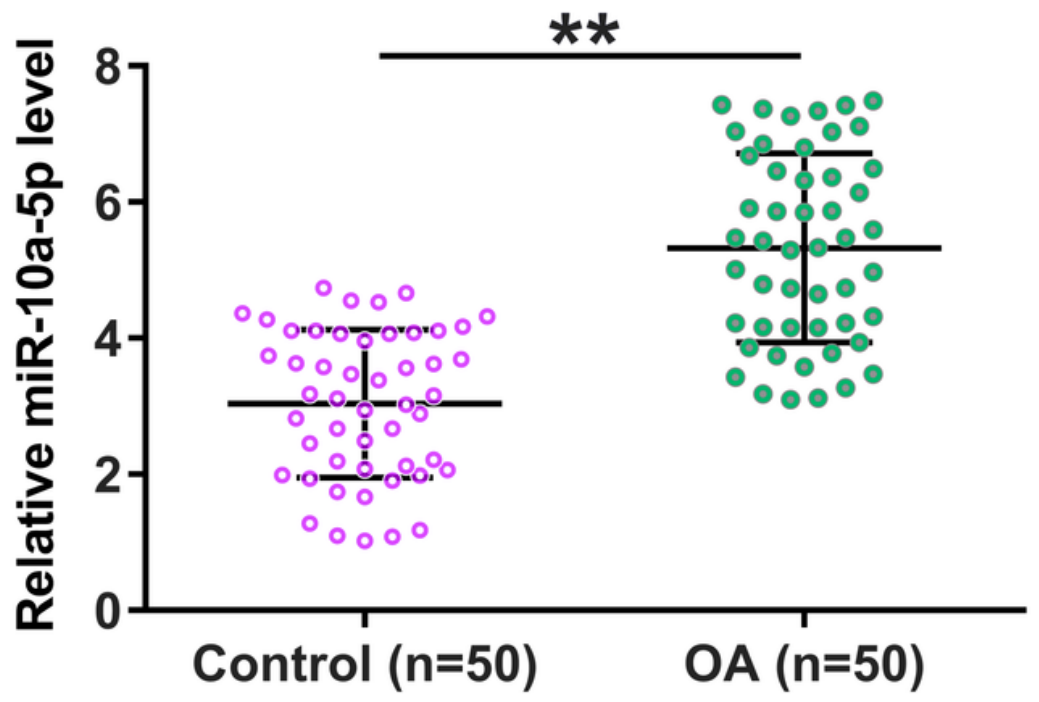

Chondrocytes

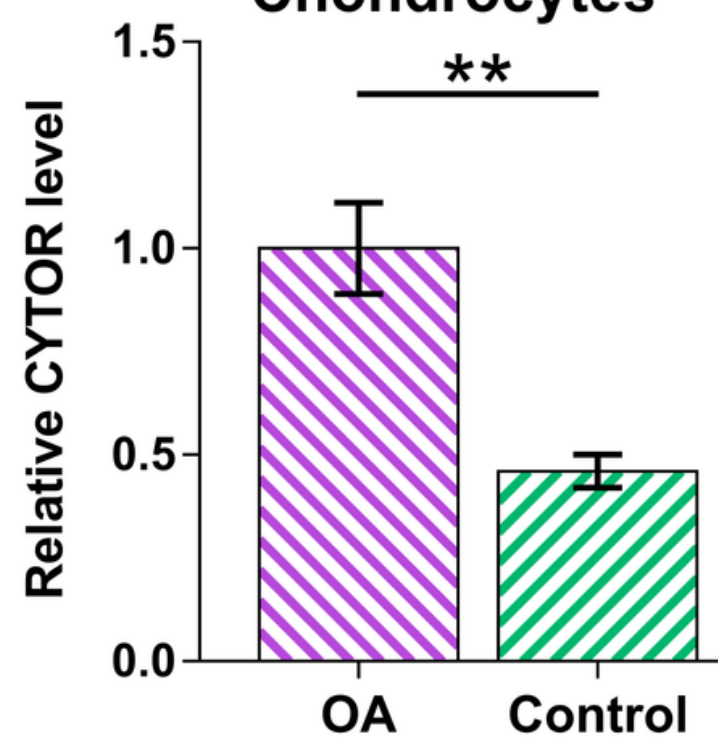

Chondrocytes

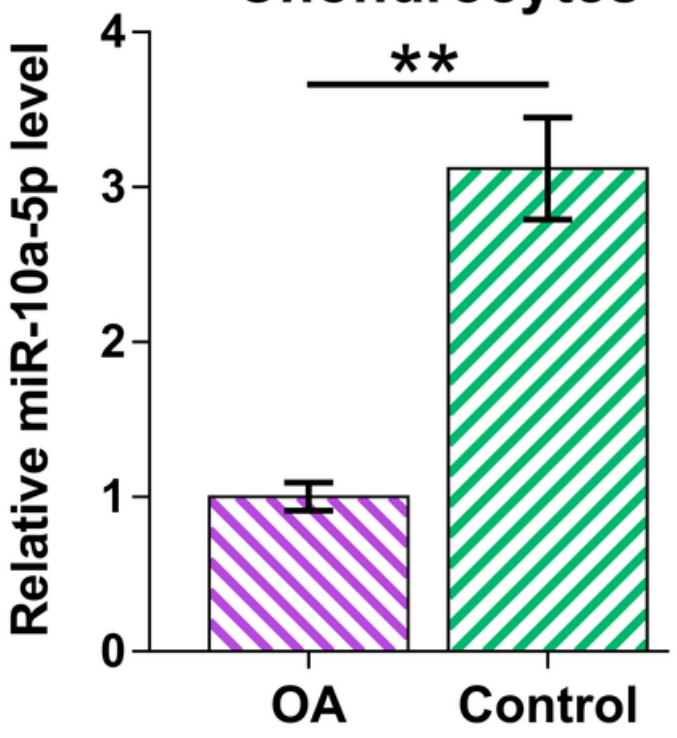

Figure 1

Expression of CYTOR and miR-10a-5p was altered in both OA patients and chondrocytes. Expression of CYTOR (A) and miR-10a-5p (B) in OA patients (synovial fluid) and chondrocytes as well as healthy patients (synovial fluid) and chondrocytes was determined by RT-qPCR. Expression data was expressed as average values of three technical replicates. ${ }^{\star *}, p<0.01$. 
Patients

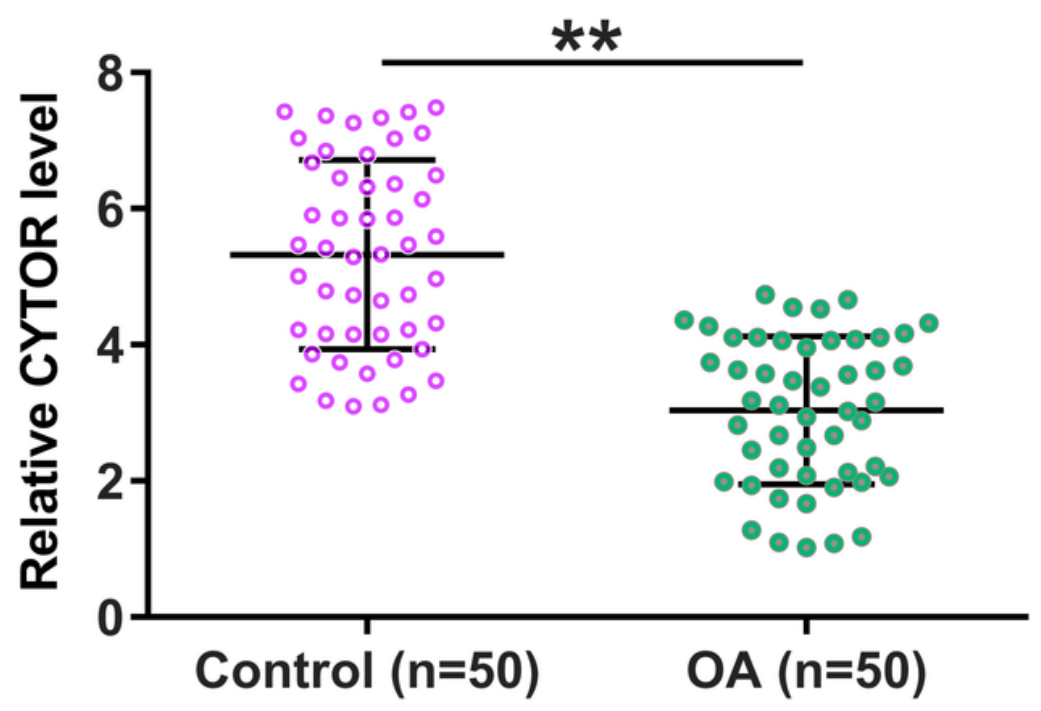

Patients

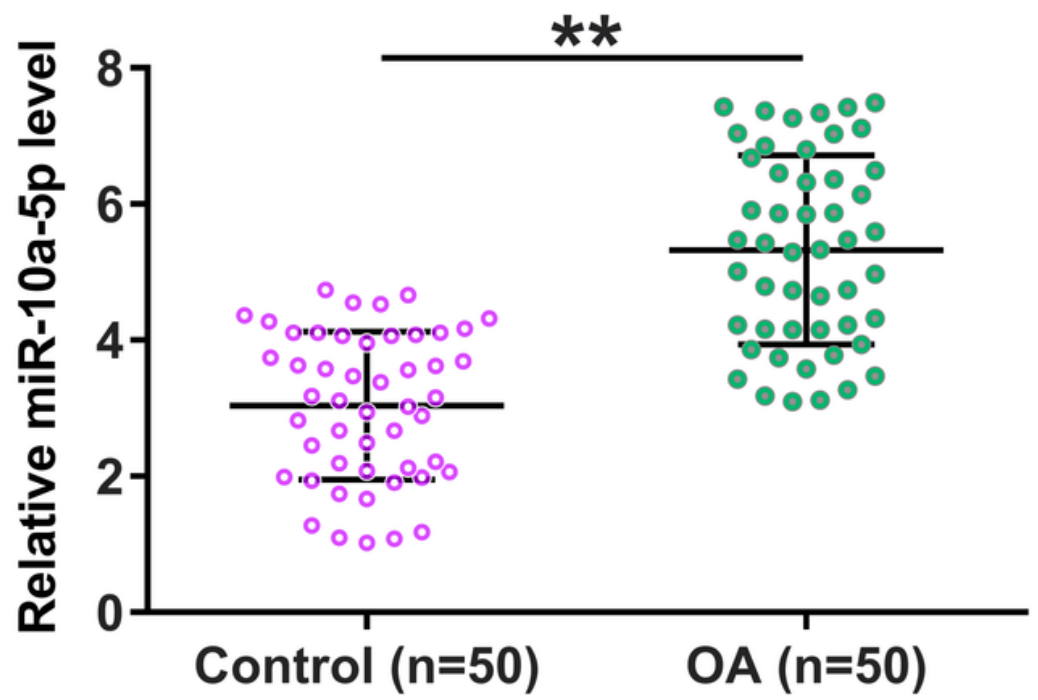

Chondrocytes

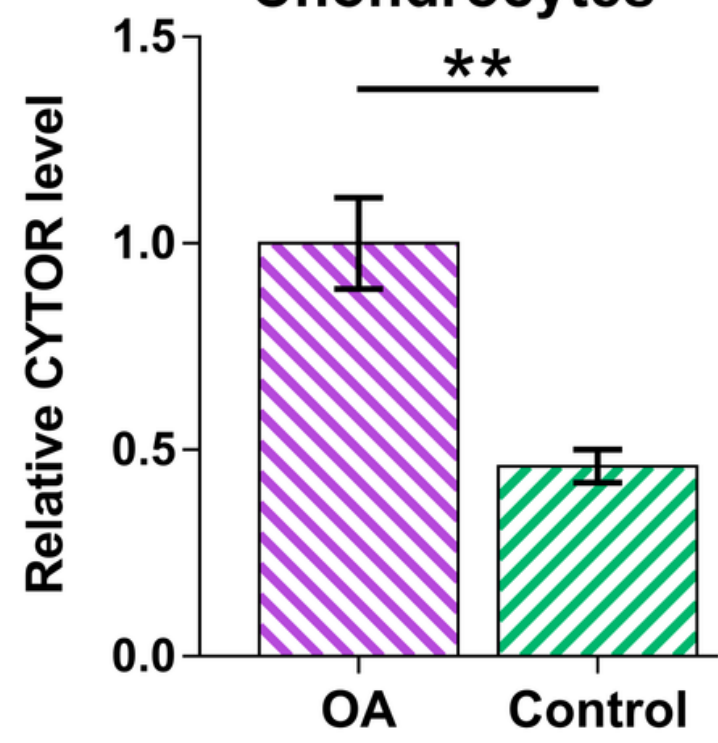

Chondrocytes

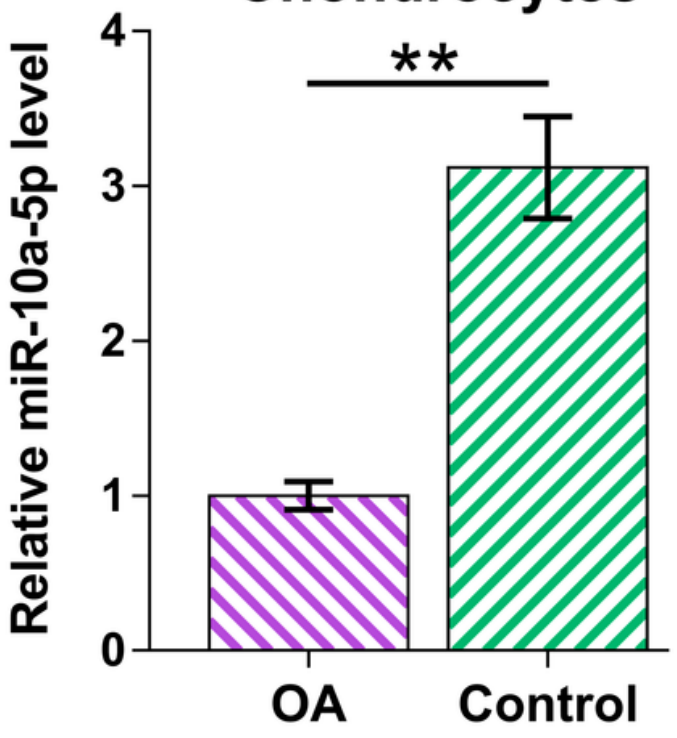

Figure 1

Expression of CYTOR and miR-10a-5p was altered in both OA patients and chondrocytes. Expression of CYTOR (A) and miR-10a-5p (B) in OA patients (synovial fluid) and chondrocytes as well as healthy patients (synovial fluid) and chondrocytes was determined by RT-qPCR. Expression data was expressed as average values of three technical replicates. ${ }^{\star *}, p<0.01$. 


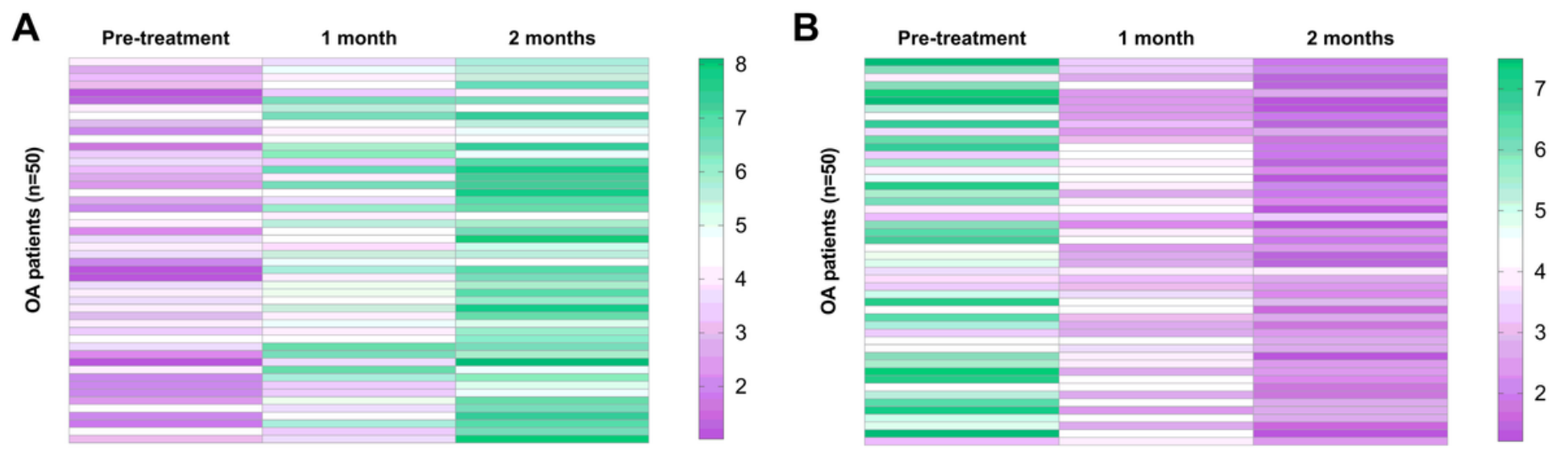

\section{Figure 2}

Treatment increased the expression of CYTOR and decreased the expression of miR-10a-5p in OA patients Expression of CYTOR (A) and miR-10a-5p (B) in synovial fluid collected from OA patients before and 1 and 2 months after the initiation of treatment. Heatmaps were plotted using Heml 1.0 software to present the changes in the expression levels of CYTOR and miR-10a-5p during treatment.

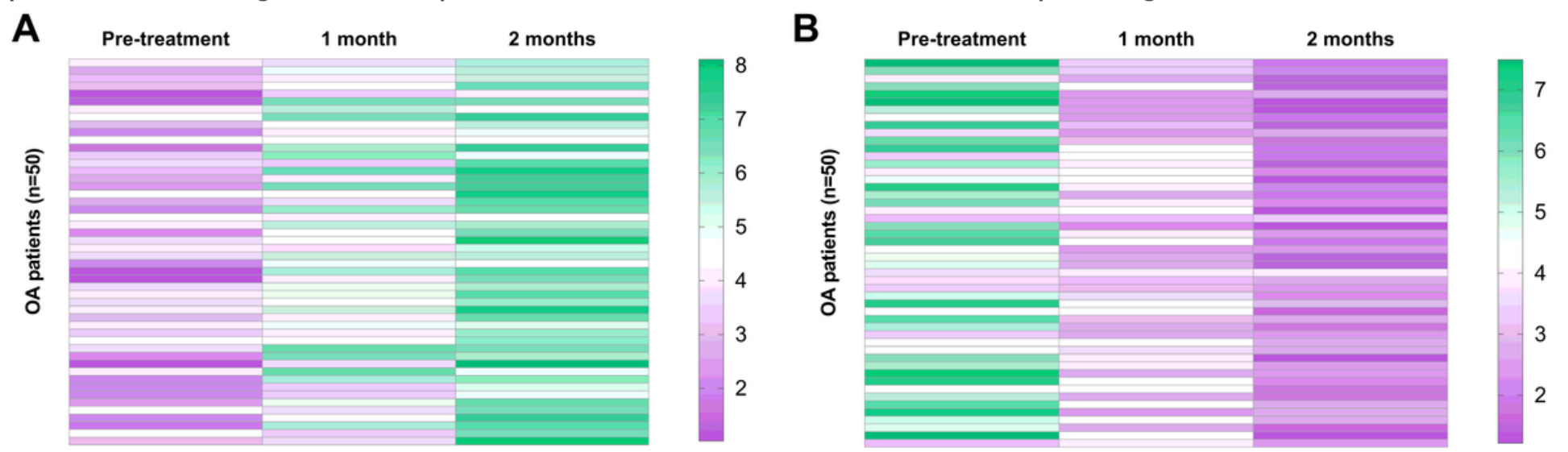

\section{Figure 2}

Treatment increased the expression of CYTOR and decreased the expression of miR-10a-5p in OA patients Expression of CYTOR (A) and miR-10a-5p (B) in synovial fluid collected from OA patients before and 1 and 2 months after the initiation of treatment. Heatmaps were plotted using Heml 1.0 software to present the changes in the expression levels of CYTOR and miR-10a-5p during treatment. 
A

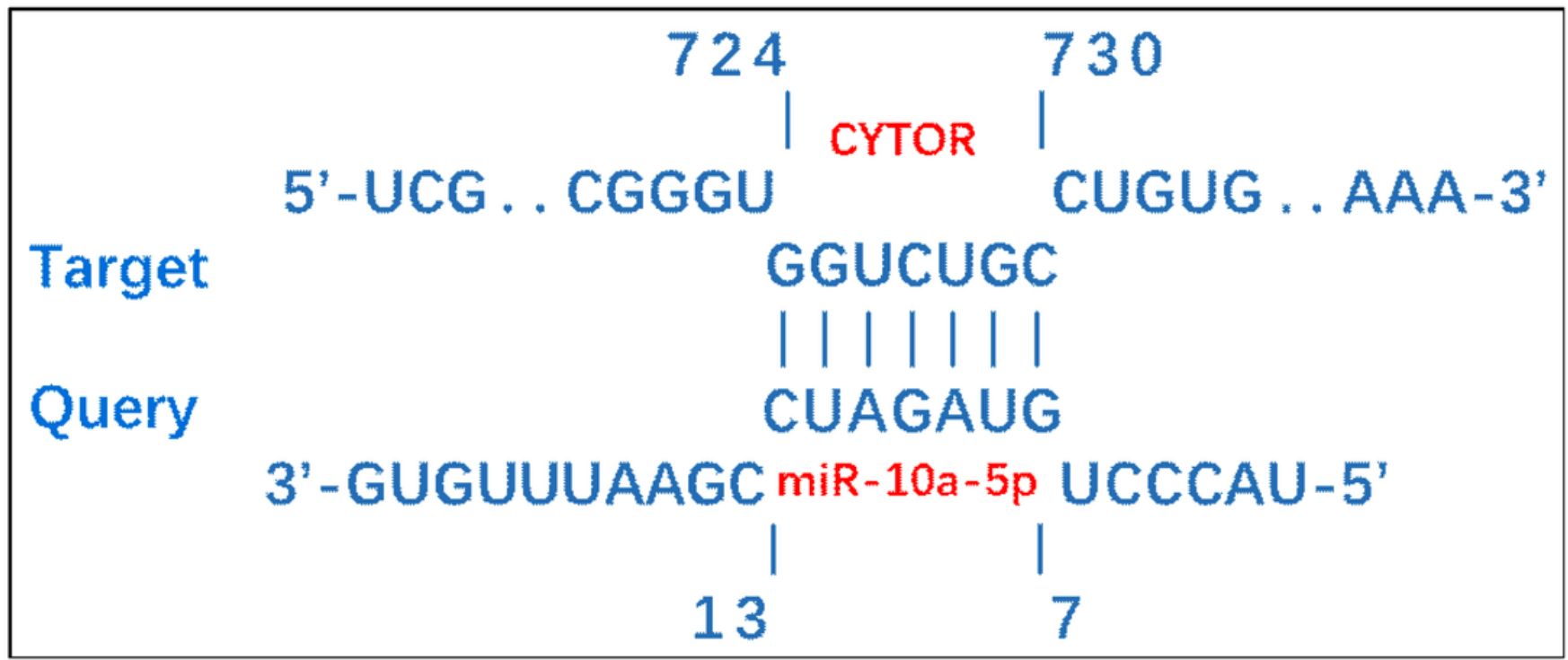

B
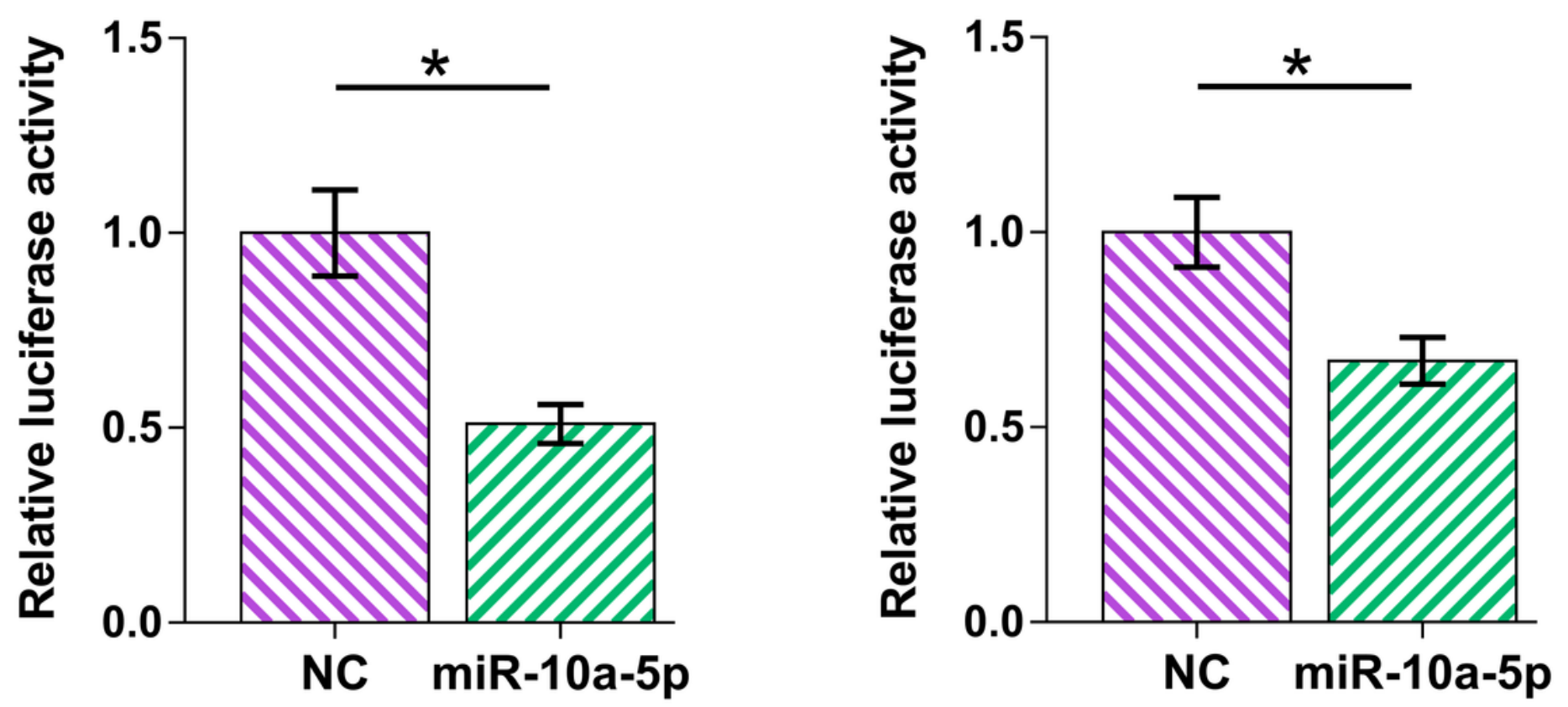

Figure 3

CYTOR and miR-10a-5p directly interacted with each other in two types of chondrocytes The potential interaction between CYTOR and miR-10a-5p was predicted by IntaRNA2.0 (A). To confirm the interaction between them, OA and control chondrocytes were co-transfected with CYTOR luciferase vector+NC miRNA (NC group) or CYTOR luciferase vector+miR-10a-5p mimic (miR-10a-5p group), luciferase activity was confirmed at $48 \mathrm{~h}$ post-transfection. ${ }^{*}, \mathrm{p}<0.05$. 
A

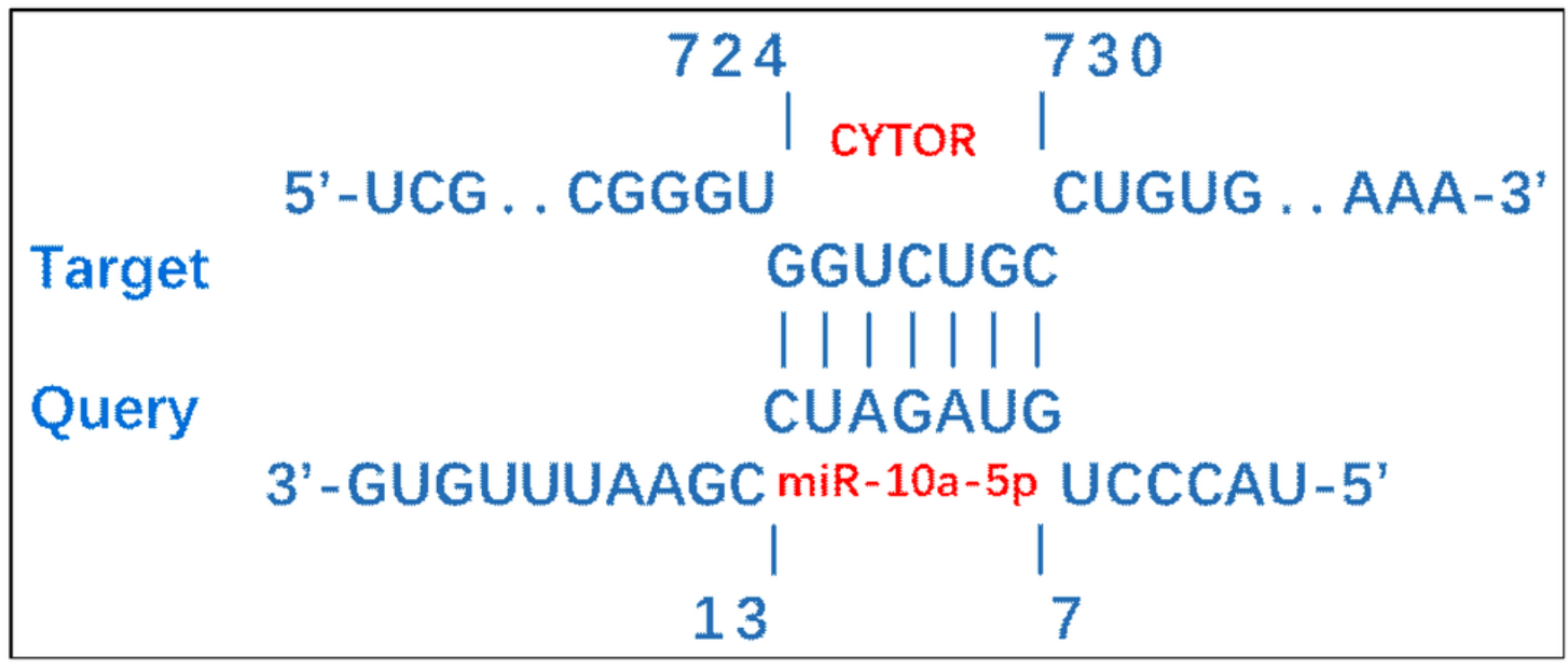

B
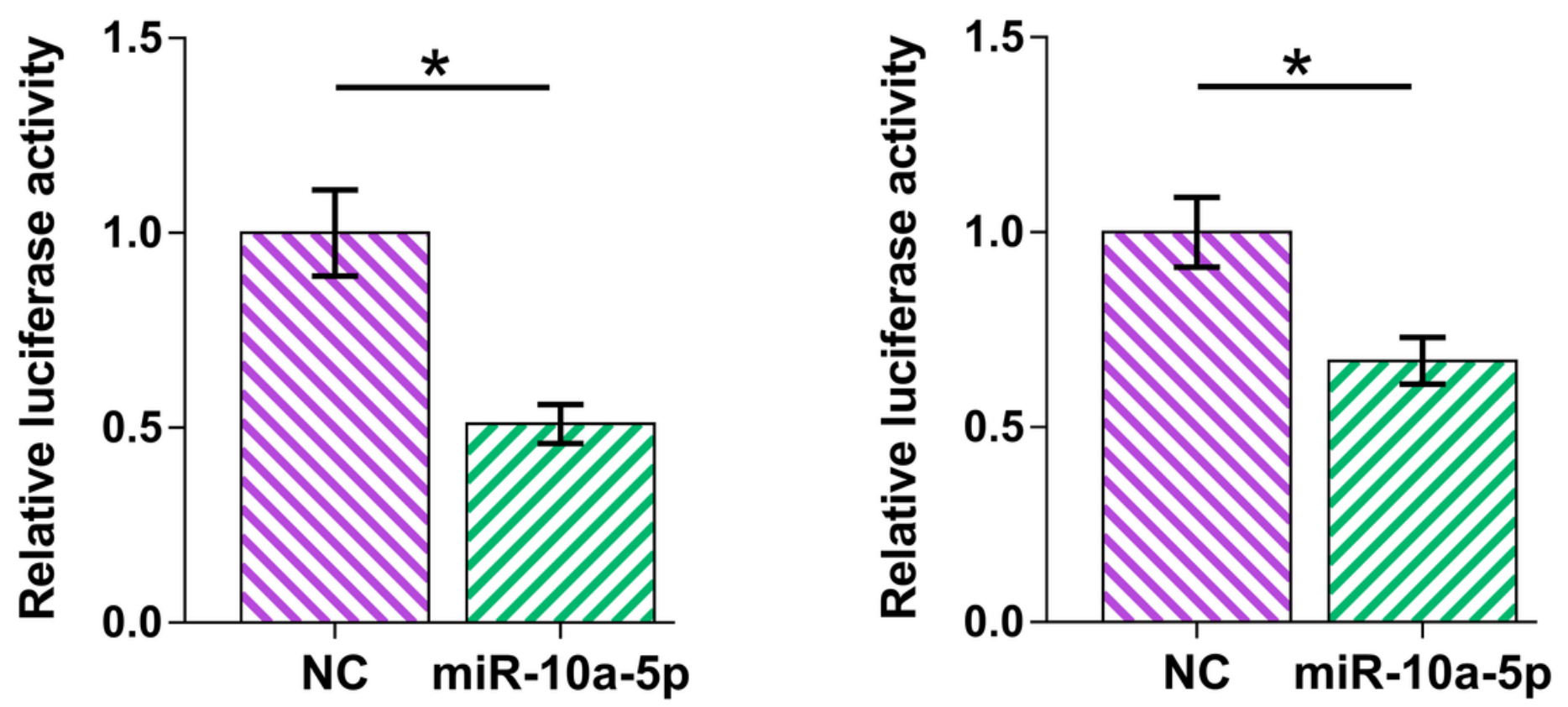

Figure 3

CYTOR and miR-10a-5p directly interacted with each other in two types of chondrocytes The potential interaction between CYTOR and miR-10a-5p was predicted by IntaRNA2.0 (A). To confirm the interaction between them, OA and control chondrocytes were co-transfected with CYTOR luciferase vector+NC miRNA (NC group) or CYTOR luciferase vector+miR-10a-5p mimic (miR-10a-5p group), luciferase activity was confirmed at $48 \mathrm{~h}$ post-transfection. ${ }^{*}, \mathrm{p}<0.05$. 

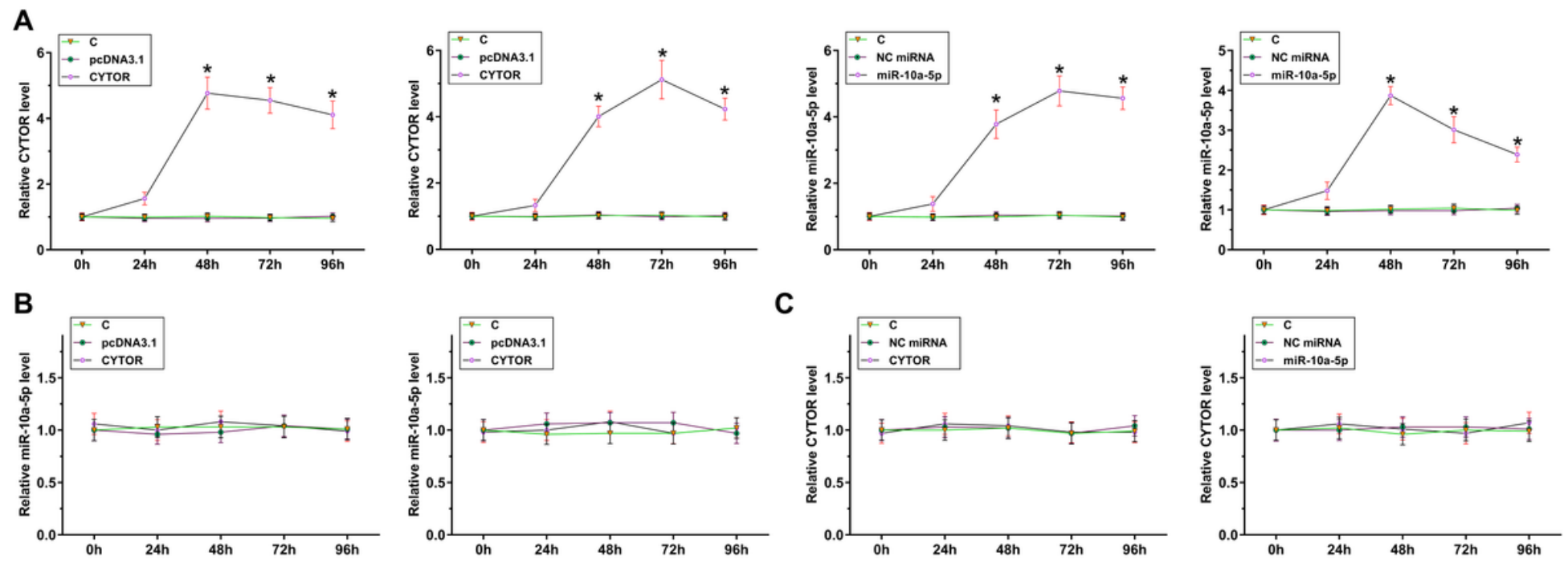

Figure 4

Overexpression of CYTOR and miR-10a-5p failed to alter the expression of each other To further explore the interaction between CYTOR and miR-10a-5p, OA and control chondrocytes were transfected with either CYTOR expression vector or miR-10a-5p mimic, and the expression of them was detected by RTqPCR every $24 \mathrm{~h}$ until $96 \mathrm{~h}(\mathrm{~A})$. The effects of the overexpression of CYTOR on miR-10a-5p (B, and the effects of the overexpression of miR-10a-5p on CYTOR (C) at different time points were also analyzed by RT-qPCR. *, compared to NC group, $p<0.05$.

A
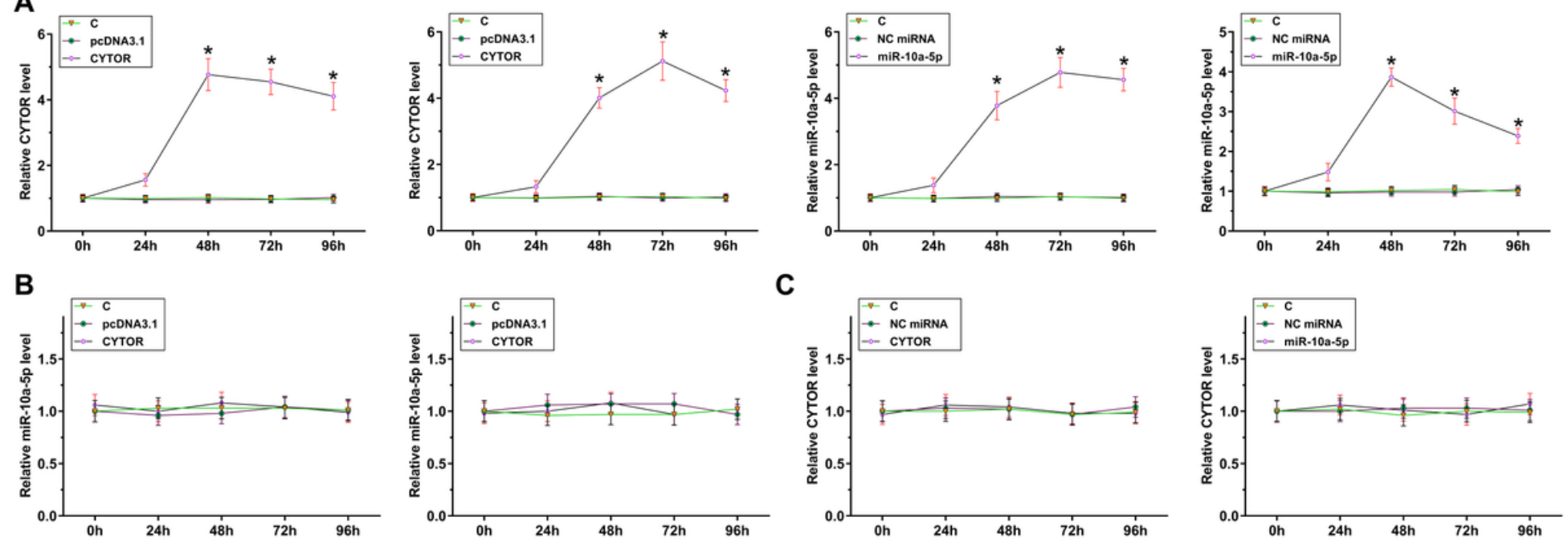

Figure 4

Overexpression of CYTOR and miR-10a-5p failed to alter the expression of each other To further explore the interaction between CYTOR and miR-10a-5p, OA and control chondrocytes were transfected with either CYTOR expression vector or miR-10a-5p mimic, and the expression of them was detected by RTqPCR every $24 \mathrm{~h}$ until $96 \mathrm{~h}(\mathrm{~A})$. The effects of the overexpression of CYTOR on miR-10a-5p (B, and the effects of the overexpression of miR-10a-5p on CYTOR (C) at different time points were also analyzed by RT-qPCR. *, compared to NC group, $p<0.05$. 


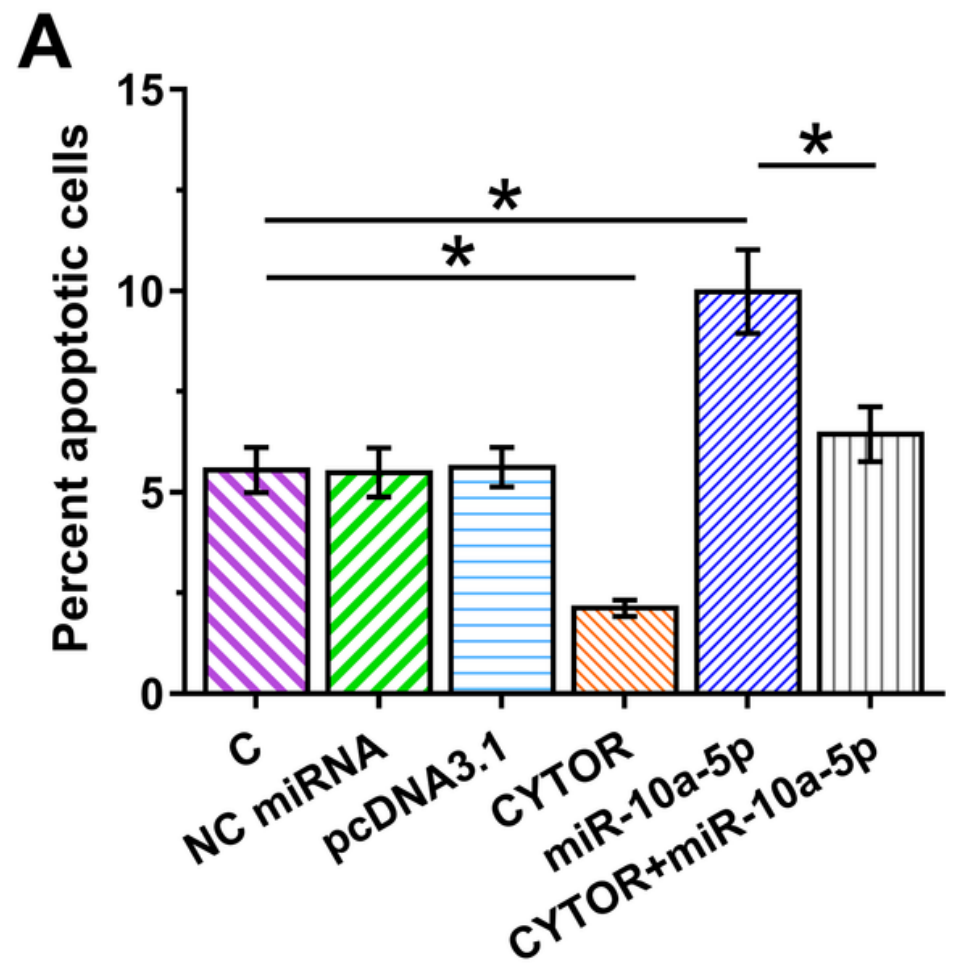

B

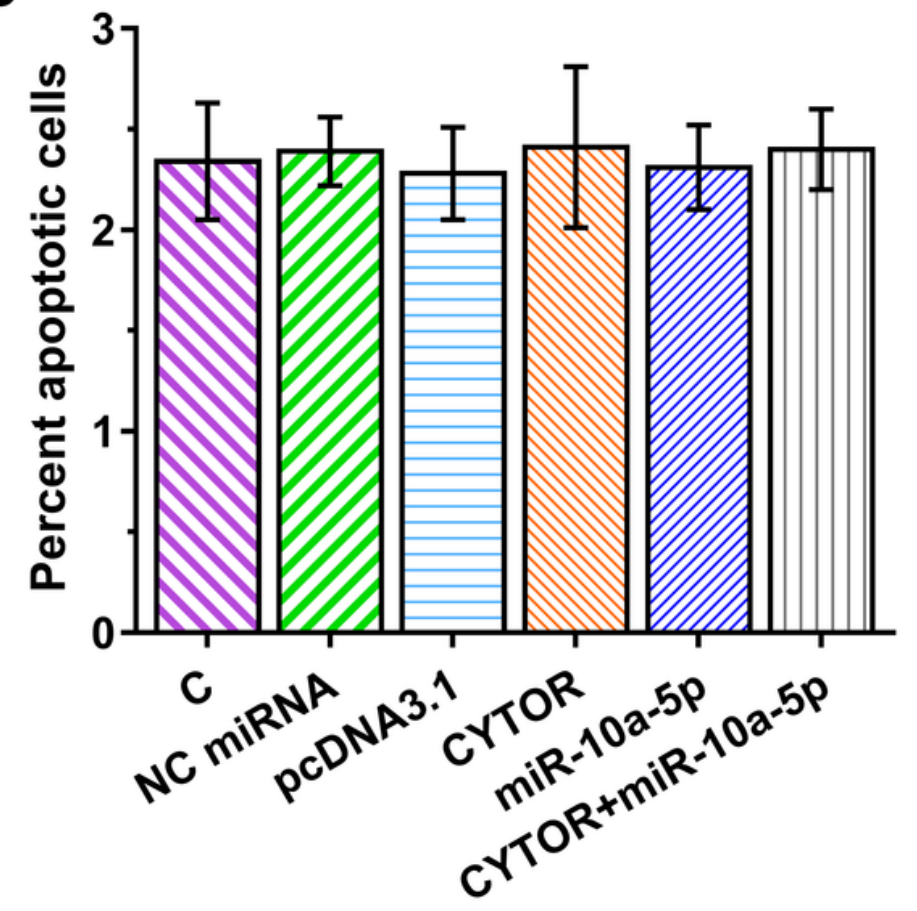

Figure 5

CYTOR overexpression suppressed the apoptosis of OA chondrocytes through miR-10a-5p The role of CYTOR and miR-10a-5p in regulating the apoptosis of OA (A) and control (B) chondrocytes was analyzed by cell apoptosis assay. *, $p<0.05$.
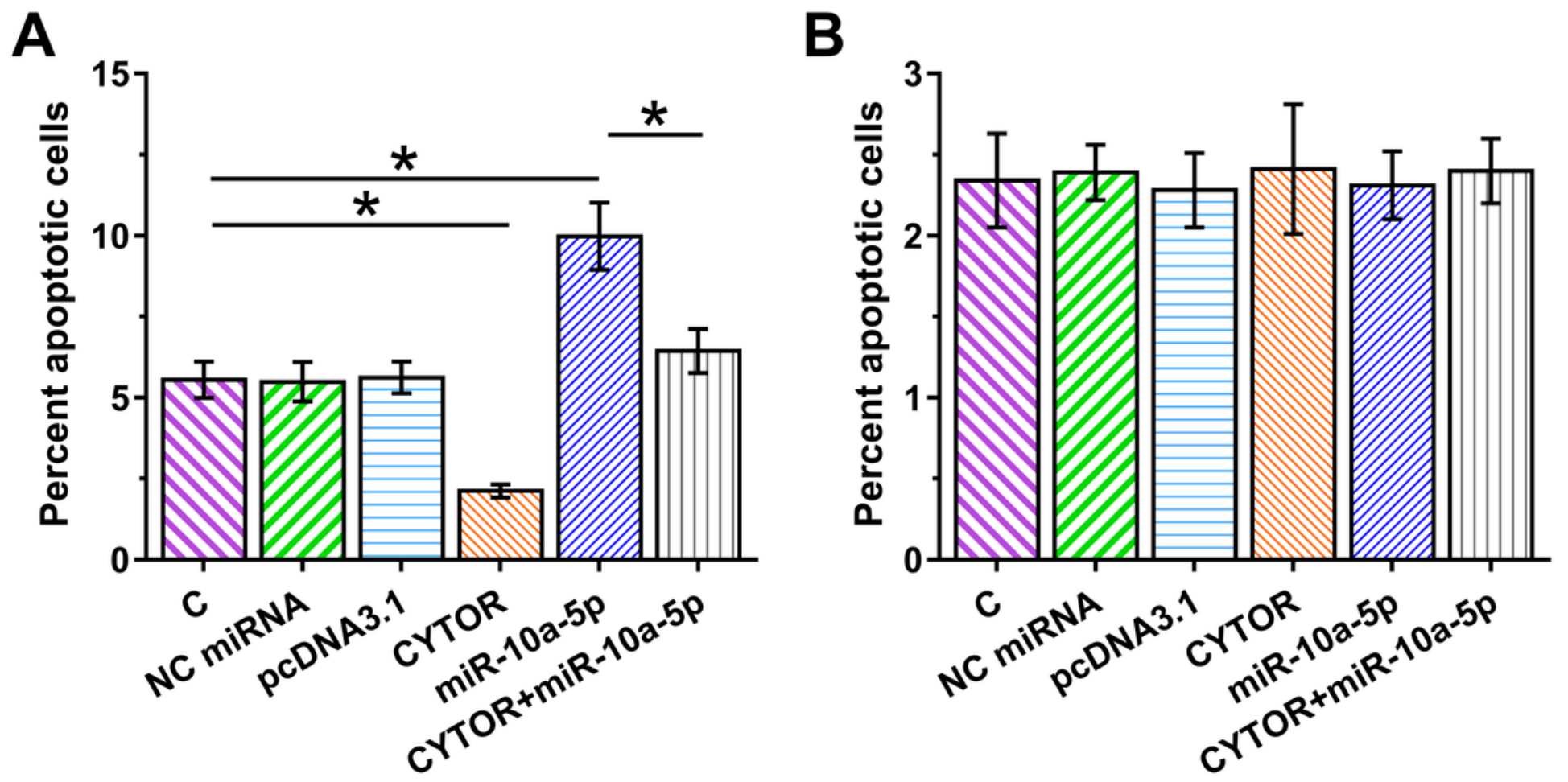

Figure 5 
CYTOR overexpression suppressed the apoptosis of OA chondrocytes through miR-10a-5p The role of CYTOR and miR-10a-5p in regulating the apoptosis of OA (A) and control (B) chondrocytes was analyzed by cell apoptosis assay. *, $p<0.05$. 